\title{
Influence of Stigma on Pre-Exposure Prophylaxis (PrEP) Care Continuum Among Men and Transwomen Who Have Sex with Men (MTWSM) in the United States
}

\author{
Jude Ssenyonjo ${ }^{1,2, *}$, Roman Shrestha ${ }^{1,2}$, Michael Copenhaver ${ }^{1,2}$ \\ ${ }^{1}$ Department of Allied Health Sciences, University of Connecticut, Storrs, CT, USA \\ ${ }^{2}$ Institute for Collaboration on Health, Intervention, and Policy, University of Connecticut, Storrs, CT, USA \\ Email address: \\ jude.ssenyonjo@uconn.edu (J. Ssenyonjo) \\ *Corresponding author
}

\section{To cite this article:}

Jude Ssenyonjo, Roman Shrestha, Michael Copenhaver. Influence of Stigma on Pre-Exposure Prophylaxis (PrEP) Care Continuum Among Men and Transwomen Who Have Sex with Men (MTWSM) in the United States. International Journal of HIV/AIDS Prevention, Education and Behavioural Science. Vol. 5, No. 2, 2019, pp. 134-140. doi: 10.11648/j.ijhpebs.20190502.18

Received: October 15, 2019; Accepted: November 9, 2019; Published: November 17, 2019

\begin{abstract}
Despite evidence from recent trials of the efficacy of pre-exposure prophylaxis (PrEP) in reducing the risk of contracting HIV, PrEP uptake has been slow due to a range of social, structural, and behavioral factors. In this systematic review, we examined the influence of stigma on the PrEP care continuum among men and transwomen who have sex with men (MTWSM). We conducted a literature search in the PubMed electronic database (2012-2018) that focused on the PrEP care continuum among high-risk MTWSM. We explored studies that specifically looked at the influence of stigma on the PrEP cascade among these socially disadvantaged populations. Our search yielded 161 articles, of which nine were ultimately included in our systematic review. The results showed a significant association between stigma and unwillingness to seek or use PrEP suggesting that stigma may negatively affect willingness and uptake of PrEP among these high-risk groups.
\end{abstract}

Keywords: Men Who Have Sex with Men, Transwomen Stigma, Pre-Exposure Prophylaxis, HIV

\section{Introduction}

\subsection{Rationale}

Men who have sex with men (MSM) account for $70 \%$ of all the HIV diagnoses [1] in the United States and are not consistently reached by existing prevention interventions. Pre-exposure prophylaxis (PrEP), an oral antiretroviral regimen taken daily by $\mathrm{HIV}$-uninfected individuals to prevent HIV acquisition, is highly efficacious in reducing HIV acquisition and could help stop the HIV epidemic among this population but is hampered by stigma. The US government established a National HIV/AIDS strategy in 2010 and set clear priorities for public health officials to refocus HIV/AIDS response to reduce new infections, increase access to care for people living with HIV, and reduce HIV related health disparities to achieve a more coordinated HIV response. This strategy is designed to reduce the current annual HIV incidence (50,000 HIV infections) by $25 \%$ within five years culminating into 163,000 infections averted with a potential saving of $\$ 48$ billion in health expenditure by the year 2020 [2]. Significant strides have been made, with a notable decline in HIV transmission across the board, except among Men and Transwomen who have Sex with Men (MTWSM [3]).

PrEP is an essential innovation in evidence-based HIV prevention for high-risk populations, including men and transwomen who have sex with men, especially with high adherence [4, 5]. The World Health Organization (WHO) and the Centers for Disease Control and Prevention (CDC) have endorsed its use, particularly among these populations. According to the $\mathrm{CDC}$, an estimated 1.2 million adults aged 18 to 59 years would benefit from PrEP in the United States, including 400,000 high-risk MTWSM [6]. Despite PrEP effectiveness in reducing the risk of HIV transmission, uptake has been slow, and focus 
has shifted toward implementation challenges and barriers to accessing and maintaining a PrEP regimen [7]. Stigma appears to be one of the significant obstacles across the overall PrEP care continuum as evidenced by a recent meta-analysis [8]. The PrEP care continuum involve five milestones: being at risk for HIV infection (e.g., sexually active MTWSM), awareness of PrEP and willingness to use it, access to PrEP services, PrEP uptake, and adherence to it [9].

Men and transwomen who have sex with men, in particular, are highly stigmatized and discriminated against due to their sexual orientation, which negatively affects health outcomes, decisions to access or utilize HIV prevention services (e.g., PrEP), and the quality of care they receive. A number of recent studies have examined factors that may affect PrEP willingness, uptake or adherence among these groups [10-24].

\subsection{Objective}

The objective of this systematic review is to summarize evidence from recent studies that have examined the influence of stigma on PrEP uptake among men and transwomen who have sex with men in the United States.

\subsection{Research Question}

Does stigma influence Pre-Exposure Prophylaxis (PrEP) Care Continuum among Men and Transwomen Who Have Sex with Men (MTWSM) in the United States?

\section{Methods}

\subsection{Search Strategy for Identification of Studies}

The literature search was conducted using the following multi-step approach. Initially research articles were identified from CINAHL, PsycINFO, LGBT Life, Global Health, Google Scholar and the PubMed electronic database using the following keywords; (barrier*[ti] OR acceptability*[ti] OR "social stigma"[MeSH] OR "social stigma" OR stigma OR stigmas OR stigmat* OR embarrass* OR fear OR fearful OR feeling*[ti] OR shame* OR discriminat* OR "negative attitude" OR "negative attitudes" OR bias OR biases OR prejudice* OR victims* OR victimize* OR stereotype*) AND ("pre-exposure prophylaxis"[MeSH] OR "prep use" OR ("pre-exposure" AND "prophylaxis") OR "pre-exposure prophylaxis" OR Truvada OR (prep AND prophylaxis)) AND (MTWSM OR gay OR homosexual* OR transgender*) NOT ("review"[PT] OR systematic [sb] OR comment [PT] OR letter [pt] OR editorial [pt] OR qualitative [ti] OR Africa* [ti] OR Thailand [ti] OR china [ti] OR Kenya [ti])

Secondly, a search for the full articles was made, and abstracts read to ensure that articles included relevant content for this study. Those that were found relevant were copied to clipboard then later exported to Endnote library for proper management and easy access. Copies of full articles were stored in a separate folder.

\subsection{Study Selection}

\subsubsection{Inclusion/Exclusion Criteria}

All articles were evaluated and only included if they met the following criteria: (1) original research study with humans, focused on HIV prevention in the context of MTWSM and PrEP use, (3) explored factors affecting willingness or uptake of PrEP including retention in care, (4) conducted between 2012-2018, and (5) stigma was mentioned as one of the barriers to uptake of any PrEPrelated services. Articles were excluded if they were animal studies, systematic reviews, were conducted outside the United States, did not focus on PrEP care continuum (e.g., willingness, linkage, retention, adherence), and were not recent.

\subsubsection{Data Extraction and Management}

Data related to the study characteristics, participant's characteristics, study design, and outcome measures were summarized from all studies included in this review using a standardized form. Article characteristics such as (a) authors, (b) study location, (c) study site, (d) sample size, (e) study population, and (f) study design were summarized. Participant characteristics included (a) age, (b) gender, (c) race/ethnicity, (d) HIV sero-status, (e) stimulant or alcohol user, and (f) engaged in transactional sex or not.

Outcome characteristics included factors influencing willingness and uptake of PrEP including; (a) knowledge about PrEP, (b) motivation to take PrEP, (c) substance/alcohol use, (d) willingness to discuss PrEP with a provider, (e) HIV risk perception, (f) self-reported condom use, and $(\mathrm{g})$ transactional sex.

\subsubsection{Search Results}

Initially, the literature search yielded 161 articles but the majority of these were either on broad HIV/AIDS topics, focused on other thematic areas and not necessarily related to the research topic, or took place outside of the United States. Our research focused on studies conducted in the United States. Since PrEP was only approved by the Food and Drug Administration (FDA) in July 2012, we only considered studies conducted between 2012 and 2018. Therefore, we excluded all studies from other countries outside of the United States $(n=13)$, those that did not have "pre-exposure prophylaxis or PrEP" within their title or abstract $(n=30)$, those that were conducted before $2012(n=12)$ and those that were not focusing on men and transwomen who have sex with men $(n=12)$. Full-text review included 18 articles of which nine (9) articles were excluded for not focusing on PrEP cascade. A total of 85 potentially relevant articles were retained. Full-text articles were retrieved, content reviewed to make sure that they fall within the study question. Reference sections were also examined to identify additional studies that may meet the inclusion criteria. Among the 85 articles, detailed review of the abstracts yielded nine (9) articles that met eligibility criteria for inclusion in the systematic review using the PRISMA guidelines as summarized in figure 1 below. 


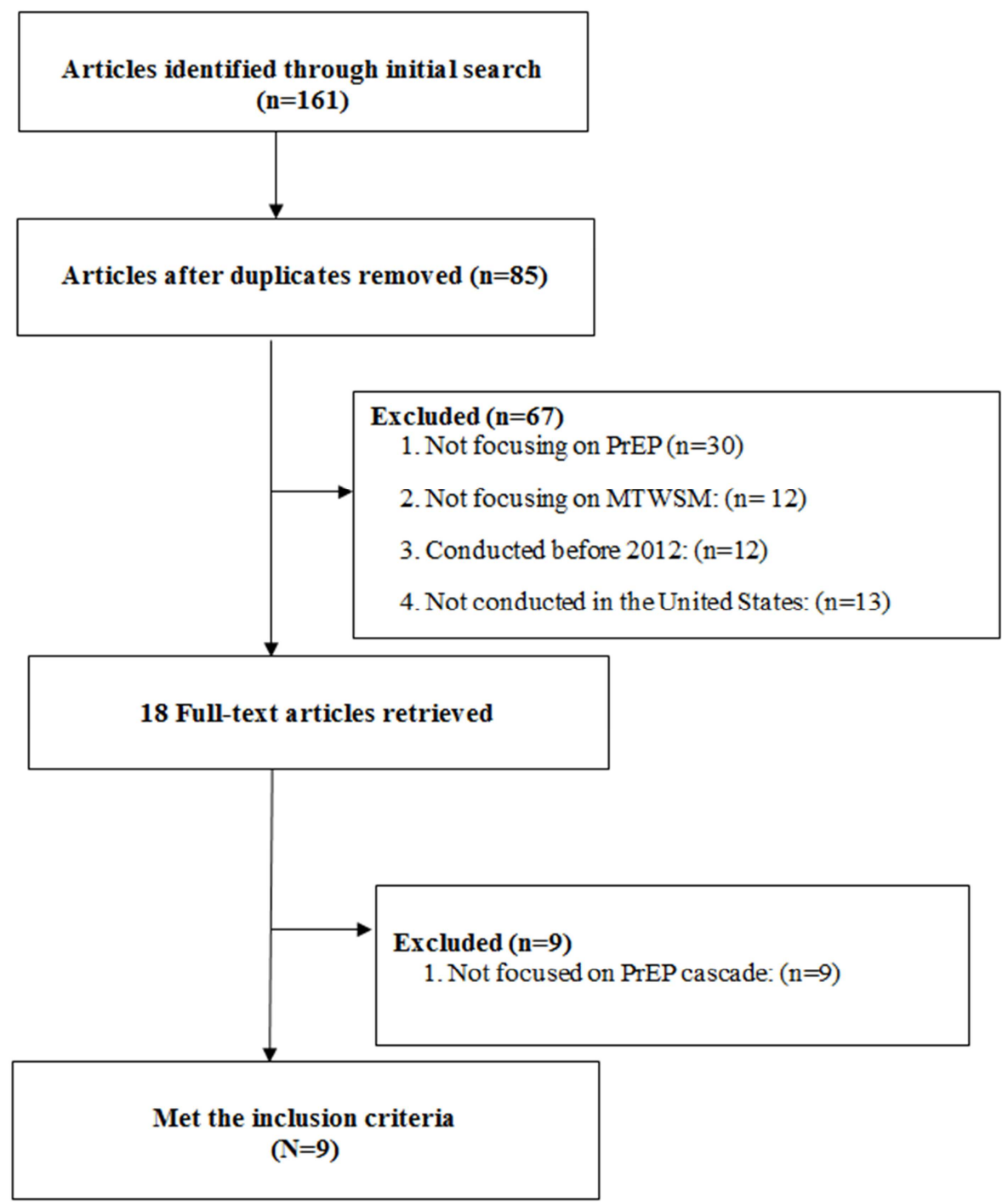

Figure 1. Literature search/study selection process flow chart.

\subsection{Study Characteristics}

A total of 9 research articles were included in this systematic review (Table 1). All the studies included information related to awareness, attitudes, stereotypes, and stigma related to the PrEP care continuum among MTWSM in general. Three studies included transgender women within their study samples; two studies compared PrEP related stigma between alcohol and stimulant-dependent MTWSM engaging in transactional sex, while one study evaluated anticipated HIV stigma related to delay in HIV testing behaviors. Furthermore, all studies that included MTWSM ensured that participants were born male regardless of current gender identity, with HIV negative sero-status (either selfreported or confirmed with an HIV antibody test), 18 years old and above, and self-reported at least one act of condomless sex (CS) with a male partner in the past 30 days.

\subsection{Social Demographic Prevalence}

All the research studies were conducted in the United States between 2011 and 2015. One of the articles did not indicate the year when the study was conducted. Two studies were conducted in Massachusetts (one in the Greater Boston area), three in New York City, one in Southeastern US, and one in San Antonio (Texas). Two of these studies were conducted through anonymous surveys, one using the largest Internet sites for MTWSM in the United States while the other was held through two booths at a Gay Pride Festival.

Table 1. Summary of studies included in the systematic review.

\begin{tabular}{lllll}
\hline Study & Study location & Study site & Sample size (N) & Study population \\
\hline Katie B. et al. & Greater-Boston area - & Private room at Fenway & \multirow{2}{*}{254} & $\begin{array}{l}\text { Substance dependent high-risk MTWSM in } \\
\text { transactional sex vs. those not in transactional sex }\end{array}$ \\
Susan A. et al. ${ }^{23}$ & Baltimore & $\begin{array}{l}\text { Health } \\
\text { Baltimore arm of NHBS, } \\
\text { BE SURE }\end{array}$ & 399 & Men who have sex with men \\
\hline
\end{tabular}




\begin{tabular}{|c|c|c|c|c|}
\hline Study & Study location & Study site & Sample size $(\mathbf{N})$ & Study population \\
\hline Sarit A et al. ${ }^{27}$ & New York City & Research Center & 160 & Men who have sex with men \\
\hline Catherine E et al. ${ }^{30}$ & Boston & $\begin{array}{l}\text { Private interview room } \\
\text { at Fenway Health }\end{array}$ & 254 & Stimulant vs. alcohol using MTWSM \\
\hline Sarit A et al..$^{32}$ & New York City & Research Center & 491 & Men who have sex with men \\
\hline Sarit A et al. ${ }^{24}$ & New York City & Research Center & 305 & $\begin{array}{l}\text { Men who have sex with men and transgender } \\
\text { women }\end{array}$ \\
\hline Moctezuma G et al. ${ }^{25}$ & San Antonio, Texas & Research Center & 159 & Men who have sex with men \\
\hline Catherine $\mathrm{E}$ et $\mathrm{al}^{31}$ & USA & Research Center & 4,098 & Men who have sex with men \\
\hline Lisa $\mathrm{A}$ et al. ${ }^{26}$ & $\begin{array}{l}\text { The Southeastern United } \\
\text { States }\end{array}$ & Gay pride festival & 285 & Men and transwomen who have sex with men \\
\hline
\end{tabular}

Table 1. Cotiuned.

\begin{tabular}{|c|c|c|}
\hline Study & Study design & Summary of findings \\
\hline Katie B. et al. ${ }^{21}$ & Cross-sectional & $\begin{array}{l}\text { No significant associations found between transactional sex and economic or healthcare-related barriers } \\
\text { to PrEP use }\end{array}$ \\
\hline Susan A. et al. ${ }^{23}$ & Cross-sectional & $\begin{array}{l}\text { Black race and perceived HIV discrimination were significantly associated with awareness and higher } \\
\text { PrEP acceptability. }\end{array}$ \\
\hline Sarit A et al. ${ }^{27}$ & $\begin{array}{l}\text { Cross-sectional } \\
\text { qualitative study }\end{array}$ & $\begin{array}{l}\text { Black and Latino MTWSM were more likely to mention HIV related stereotypes and significantly less } \\
\text { likely to mention promiscuity than white MTWSM. }\end{array}$ \\
\hline $\begin{array}{l}\text { Catherine E et } \\
\text { al. }\end{array}$ & $\begin{array}{l}\text { Cross-sectional } \\
\text { quantitative assessment }\end{array}$ & $\begin{array}{l}\text { Stimulant using MTWSM were more likely to be concerned about substance use affecting PrEP } \\
\text { adherence than alcohol using MTWSM. Similarly, stimulant using MTWSM were less concerned about } \\
\text { HIV stigma as a barrier to PrEP uptake than alcohol users. }\end{array}$ \\
\hline Sarit A et al. ${ }^{32}$ & Cross-sectional & $\begin{array}{l}\text { Black Latino MTWSM were more likely to consider talking to a doctor about their sex life as a barrier to } \\
\text { PrEP uptake and were less likely to endorse agency in medical decision-making. }\end{array}$ \\
\hline Sarit A et al. ${ }^{24}$ & Cross-sectional & Anticipated HIV stigma was strongly associated with decreased HIV testing. \\
\hline $\begin{array}{l}\text { Moctezuma G et } \\
\text { al. }^{25}\end{array}$ & Cross-sectional & Overall, there is a significant association between PrEP awareness and age, education and income levels. \\
\hline $\begin{array}{l}\text { Catherine E et } \\
\text { al. }^{31}\end{array}$ & Cross-sectional & $\begin{array}{l}\text { There is an association between lower structural stigma and decreased odds of condomless anal } \\
\text { intercourse and increased odds of having heard of or taken PEP and PrEP. }\end{array}$ \\
\hline Lisa A et al. ${ }^{26}$ & Cross-sectional & There is a strong association between PrEP being related to promiscuity and lack of interest in PrEP use. \\
\hline
\end{tabular}

\subsection{Study Measures}

The most common measures included were social demographic characteristics (8/10), followed by knowledge of PrEP, willingness to take PrEP, and sexual behaviors (4/10), PrEP utilization and interest (3/10), alcohol and stimulant dependence, hypothetical barriers to PrEP use, HIV testing behavior, barriers to PrEP access and uptake and access to health care each (2/10). Transactional sex, sexually transmitted infection (STI) history, HIV risk prevention behaviors, HIV stigma, PrEP awareness, state-level HIV prevalence, state-level structural stigma, and PrEP stigma were the least common measures used in studies $(1 / 10)$. A cumulative total of 6,384 MTWSM were interviewed across the nine studies.

Although our primary objective was to examine the influence of stigma on PrEP care continuum among MTWSM, we did not find studies that looked at the influence of stigma on later stages of PrEP cascade. Therefore, our findings are not structured based on overall PrEP care continuum. Instead, we report our results based on individual level and structural level barriers that influence decisions to take PrEP.

\section{Results}

\subsection{Individual-level Barriers to Seeking PrEP}

Biello et al. (2017) found an association between patients' concern that providers would negatively judge their sexual behaviors and patients' preference to seek PrEP from providers other than the primary care provider [12]. MTWSM who recently engaged in transactional sex often reported barriers related to; need to conceal PrEP use from their partners, fear that PrEP use would negatively affect their sex life with their primary partners if they found out about it, and fear that the primary partner would think they were infected due to PrEP use. In comparison with other high-risk, substance-dependent MTWSM, MTWSM in transactional sex was more likely to report concerns regarding PrEP use in fear of both casual and main partners' knowledge of use [12]. However, stimulant and alcohol using MTWSM in another study expressed different perceptions about barriers to PrEP use. Whereas stimulant using MTWSM were more concerned about substance use as a barrier to uptake of PrEP, alcohol using MTWSM cited HIV stigma as a significant limiting factor in their use of PrEP [22]. Surprisingly, neither study found substantial differences in structural or individual-level barriers to PrEP use between MTWSM who engaged in transactional sex and those who did not $[12,22]$.

Fallon et al. (2017) found out that HIV-related stigma could negatively influence attitudes about PrEP use, and perceived stigma and discrimination were shown to have a significant impact on PrEP awareness and acceptability [16]. Similarly, negative stereotypes about PrEP use were predominantly identified by most of the study participants in another study by Golub et al. Stereotypes such as assuming that PrEP users are HIV positive individuals who are resistant 
to condom use and promiscuous negatively impact willingness to discuss PrEP use with sexual partners or health providers [19, 25]. Garcia et al. (2017) found that the majority of Latino MTWSM expressed concern that taking PrEP would lead to others' perceptions of them as gay and the resulting stigma. Taking PrEP was related to being a person living with HIV (PLHIV), and promiscuity [18]. Further, Garcia et al. (2017) found that black Latino MTWSM were significantly more likely to endorse stigmarelated concerns regarding PrEP, specifically others' perceptions of why they are taking the pill or assuming they are HIV positive if they are seen taking the medicine. Daily pill intake was predominantly a concern for Black and Latino MTWSM than for other demographics in the sample [18]. The additional evidence is provided by Eaton et al. where the majority of study participants believed that PrEP was intended for promiscuous individuals, which was strongly associated with a lack of interest in using PrEP [15].

It should be noted that the fear of testing HIV positive also raises stigma. Golub et al. (2017) found that individuals who had not been tested in the previous six months reported significantly higher anticipated stigma scores than those who had tested for HIV recently. Anticipated HIV stigma was strongly associated with decreased odds of HIV testing. However, there is no evidence of this finding from other studies due to the different measures used [20].

HIV risk perception is another critical barrier that affects PrEP uptake fueled by alcohol and stimulant use. Stimulant using MTWSM were more likely to engage in condomless sex with HIV-infected or partners with unknown HIV status compared to alcohol users [22].

\subsection{Structural Level Stigma-related Barriers}

There is evidence that less supportive environments for Lesbian, Gay, Bisexual, and Transgender (LGBT) people were significantly associated with increased sexual risk behavior, decreased awareness and PrEP use, as well as reduced comfort discussing sexual behavior with primary care providers among HIV uninfected MTWSM in the United States. MTWSM living in states that do not recognize same-sex relationships, have public high schools without a safe and supportive environment for the lesbian, gay, bisexual, and transgender (LGBT) community, and having no state policies or laws related to sexual orientation discrimination (including legalized marriage for same-sex couples, employment non-discrimination laws, protections against hate crimes, and legality of joint adoption for samesex couples) are less likely to report having engaged in discussion with their provider's issues related to having sex with men, condomless sex, and HIV prevention strategies [23].

\section{Discussion}

We found evidence of the significant influence of stigma on PrEP care continuum among MTWSM. Stigma related to $\mathrm{HIV}$ and sexual promiscuity is a known barrier to PrEP use
$[26,27]$. Evidence from this study indicates that participants reported fear of being stigmatized by providers and their sexual partners if they expressed interest in using PrEP. Perceived stigma and negative stereotypes such as linking PrEP users to being HIV positive, promiscuous and gay negatively impact willingness to discuss PrEP use with sexual partners or providers and led to majority opt to conceal PrEP use from their sexual partners. This finding indicates that HIV related stigma has a significant impact on PrEP awareness and use among MTWSM and other high-risk populations who could benefit from PrEP intervention.

The results of our study appear to be consistent with findings from other populations, such as women. A recent study that examined the pervasiveness of PrEP stigma among US women found that majority perceived PrEP-user stereotypes, such as belief that others would regard them as promiscuous (37\%), HIV-positive (32\%), bad (14\%), gay $(11 \%)$ if they used PrEP $[27,26]$. All studies in this review suggest a significant influence of stigma on the desire to seek or use PrEP.

It is therefore imperative for HIV prevention programs to devise specific strategies to address potential sources of stigma, both at individual and structural levels if PrEP programs are to succeed. The structural level stigma emanating from providers' negative attitudes towards MTWSM, and unwillingness to prescribe PrEP suggests a lesser conducive environment for patients to freely discuss HIV preventions strategies with their primary care providers. Provider's negative attitudes are significant barrier that needs to be addressed if we are to achieve HIV prevention targets among this high-risk population.

Furthermore, this finding is consistent with other studies conducted among similar populations outside the U.S. for example, a qualitative study conducted among adult transwomen in Rio de Janeiro, Brazil identified past experiences of transgender-identity related discrimination in the universal health care system as the most prominent barrier to PrEP uptake. Similarly, $64 \%$ of participants recalled at least one stigmatizing interaction with a health care provider in a recent study that examined the experiences of gay men who adopted PrEP.

The relatively homophobic environment in the United States may have played a role in creating a non-supportive environment for MTWSM. A significant association was found between states with less support for same-sex relationships, having no state policies and laws related to sexual orientation discrimination and increased sexual risk behavior, decreased PrEP awareness and use [23].

\section{Future Implications}

Findings of this study indicate that stigma among MTWSM is still a widespread problem in the United States that needs to be addressed urgently if HIV is to be averted among this population. There is a need for an environment which enables Men and Transwomen to freely discuss their health concerns with health providers 
about PrEP and other behavioral prevention strategies. Since most MTWSM experience or anticipate stigmatizing attitudes from family, friends and sexual partners, their ability to use PrEP is limited due to stigma. Establishing support groups may be a feasible and successful platform for experience sharing among those who have faced similar challenges but managed to successfully enroll in PrEP programs, and those who are struggling to access PrEP due to anticipated stigma.

A social media-based approach could potentially be utilized to identify, reach high-risk MTWSM with HIV prevention messages, foster risk perception, create demand for PrEP and linking individuals to sites where they can receive PrEP. This approach can also be used to address stigma related to PrEP. The other strategy that can potentially be utilized to reduce stigma is the use of mobile phones and other wireless devices (mHealth) to provide PrEP information, online enrollment. MTWSM can use these mobile devices for self-risk assessment and enroll in PrEP care without face-to face contact with providers. The PrEP prescription can be done online and delivered to the individual's address. An alternative arrangement is possible for homeless MTWSM to acquire PrEP through peer support groups.

Stigma from friends, family members, and sexual partners can be addressed through increased awareness using digital media and health psychologists deployed at PrEP provider points. Call centers or a hotline can also be established to provide online counseling to those MTWSM with stigma related challenges and used to link those who may want to enroll in PrEP care.

\section{Study Limitations}

This systematic review has some limitations that should be acknowledged. First, our extensive literature search did not reveal any studies that assessed the influence of stigma on adherence to PrEP and long-term engagement in PrEP services. We, therefore, we're unable to capture the influence of stigma over each stage of PrEP care continuum. Secondly, studies that were examined assessed hypothetical barriers to PrEP utilization. Measuring willingness to PrEP uptake using hypothetical scenarios may not correlate with actual intentions to use PrEP. Thirdly, behavioral data collected in these studies were self-reported, which means we cannot rule out variations due to differences in recall or social desirability bias, especially when applied to stigmatized behaviors and PrEP acceptability, and this may result in either overestimation or underestimation. This may have also led to the inclusion of HIV positive MTWSM in the study who felt uncomfortable disclosing their true HIV sero-status due to stigma. Lastly, the data used in most of the studies were cross-sectional, which prevents causal relationships to be examined. A short study period cannot provide enough evidence on awareness and willingness to use PrEP as attitudes and perceptions change rapidly with increasing availability and press coverage. There could also be issues of generalizability due to the studies having focused on particular regions as opposed to all the states in the US.

\section{Conclusion}

Our study presents significant findings that support the need to address PrEP related stigma stemming from homophobia and other forms of negative attitudes from families, communities, providers and sexual partners of men and transwomen who have sex with men. Stigma poses significant implications for HIV prevention efforts among high-risk populations. The evidence summarized in this review highlights the need to develop tailored strategies to specifically address stigma at the individual level as well as at the structural level if PrEP interventions are to yield better outcomes. It is possible that future PrEP users will have different perceptions and experiences due to increasing knowledge of PrEP and exposure to PrEP interventions. Further research is necessary to gain perspectives from other communities or high risk groups currently using or those who may potentially benefit from PrEP in a different social cultural context.

\section{Conflicts of Interest}

All the authors do not have any possible conflicts of interest.

\section{Acknowledgements}

This work was supported by grants from the National Institute on Drug Abuse for career development (K02 DA033139) to Michael Copenhaver and the National Institute of Mental Health Research Training Grant T32MH074387.

\section{References}

[1] CDC. HIV/AIDS Basic Statistics. 2017 December 18, 2017 [cited 2018 April 9, 2018]; HIV/AIDS Statistics]. Available from: https://www.cdc.gov/hiv/basics/statistics.html.

[2] Holtgrave, D. R., et al., Costs, consequences and feasibility of strategies for achieving the goals of the National HIV/AIDS strategy in the United States: a closing window for success? AIDS Behav, 2012. 16 (6): p. 1365-72.

[3] Singh, S., A. Mitsch, and B. Wu, HIV Care Outcomes Among Men Who Have Sex With Men With Diagnosed HIV InfectionUnited States, 2015. MMWR Morb Mortal Wkly Rep, 2017. 66 (37): p. 969-974.

[4] Anderson, P. L., et al., Emtricitabine-tenofovir concentrations and pre-exposure prophylaxis efficacy in men who have sex with men. Sci Transl Med, 2012. 4 (151): p. 151ra125.

[5] Grant, R. M., et al., Uptake of pre-exposure prophylaxis, sexual practices, and HIV incidence in men and transgender women who have sex with men: a cohort study. Lancet Infect Dis, 2014. 14 (9): p. 820-9. 
[6] Smith, D. K., et al., Vital Signs: Estimated Percentages and Numbers of Adults with Indications for Preexposure Prophylaxis to Prevent HIV Acquisition--United States, 2015. MMWR Morb Mortal Wkly Rep, 2015. 64 (46): p. 1291-5.

[7] Marcus, J. L., et al., Successful Implementation of HIV Preexposure Prophylaxis: Lessons Learned From Three Clinical Settings. Curr HIV/AIDS Rep, 2016. 13 (2): p. 11624.

[8] Peng, P., et al., A Global Estimate of the Acceptability of Preexposure Prophylaxis for HIV Among Men Who have Sex with Men: A Systematic Review and Meta-analysis. AIDS Behav, 2018. 22 (4): p. 1063-1074.

[9] Kelley, C. F., et al., Applying a PrEP Continuum of Care for Men Who Have Sex With Men in Atlanta, Georgia. Clin Infect Dis, 2015. 61 (10): p. 1590-7.

[10] Adams, J. W., J. Shinefeld, and K. A. Brady, Acceptability of Oral Preexposure Prophylaxis Among Men Who Have Sex With Men in Philadelphia. J Acquir Immune Defic Syndr, 2016. 73 (3): p. e62-e65.

[11] Arnold, T., et al., Social, structural, behavioral and clinical factors influencing retention in Pre-Exposure Prophylaxis (PrEP) care in Mississippi. PLoS One, 2017. 12 (2): p. $\mathrm{e} 0172354$.

[12] Biello, K. B., et al., The Safe Sex"Conundrum: Anticipated Stigma From Sexual Partners as a Barrier to PrEP Use Among Substance Using MSM Engaging in Transactional Sex. AIDS Behav, 2017. 21 (1): p. 300-306.

[13] Bil, J. P., et al., The Acceptability of Pre-Exposure Prophylaxis: Beliefs of Health-Care Professionals Working in Sexually Transmitted Infections Clinics and HIV Treatment Centers. Front Public Health, 2018. 6: p. 5.

[14] Calabrese, S. K., et al., The impact of patient race on clinical decisions related to prescribing HIV pre-exposure prophylaxis (PrEP): assumptions about sexual risk compensation and implications for access. AIDS Behav, 2014. 18 (2): p. 226-40.

[15] Eaton, L. A., et al., Stigma and Conspiracy Beliefs Related to Pre-exposure Prophylaxis (PrEP) and Interest in Using PrEP Among Black and White Men and Transgender Women Who Have Sex with Men. AIDS Behav, 2017. 21 (5): p. 1236-1246.

[16] Fallon, S. A., et al., Awareness and Acceptability of Preexposure HIV Prophylaxis Among Men Who have Sex with Men in Baltimore. AIDS Behav, 2017. 21 (5): p. 1268-1277.
[17] Garcia, J., et al., Psychosocial Implications of Homophobia and HIV Stigma in Social Support Networks: Insights for High-Impact HIV Prevention Among Black Men Who Have Sex With Men. Health Educ Behav, 2016. 43 (2): p. 217-25.

[18] Garcia, M. and A. L. Harris, PrEP awareness and decisionmaking for Latino MSM in San Antonio, Texas. PLoS One, 2017. 12 (9): p. e0184014.

[19] Golub, S. A. and K. E. Gamarel, The impact of anticipated HIV stigma on delays in HIV testing behaviors: findings from a community-based sample of men who have sex with men and transgender women in New York City. AIDS Patient Care STDS, 2013. 27 (11): p. 621-7.

[20] Golub, S. A., K. E. Gamarel, and A. Surace, Demographic Differences in PrEP-Related Stereotypes: Implications for Implementation. AIDS Behav, 2017. 21 (5): p. 1229-1235.

[21] Holloway, I. W., et al., Facilitators and Barriers to PreExposure Prophylaxis Willingness Among Young Men Who Have Sex with Men Who Use Geosocial Networking Applications in California. AIDS Patient Care STDS, 2017. 31 (12): p. 517-527.

[22] Oldenburg, C. E., et al., Differences in Attitudes About HIV Pre-Exposure Prophylaxis Use Among Stimulant Versus Alcohol Using Men Who Have Sex with Men. AIDS Behav, 2016. 20 (7): p. 1451-60.

[23] Oldenburg, C. E., et al., State-level structural sexual stigma and HIV prevention in a national online sample of HIVuninfected MSM in the United States. Aids, 2015. 29 (7): p. 837-45.

[24] Perez-Figueroa, R. E., et al., Acceptability of PrEP Uptake Among Racially/Ethnically Diverse Young Men Who Have Sex With Men: The P18 Study. AIDS Educ Prev, 2015. 27 (2): p. 112-25.

[25] Franks, J., et al., Sex, PrEP, and Stigma: Experiences with HIV Pre-exposure Prophylaxis Among New York City MSM Participating in the HPTN 067/ADAPT Study. AIDS Behav, 2018. 22 (4): p. 1139-1149.

[26] Calabrese, S. K., et al., HIV Pre-Exposure Prophylaxis Stigma as a Multidimensional Barrier to Uptake Among Women Who Attend Planned Parenthood. J Acquir Immune Defic Syndr, 2018. 79 (1): p. 46-53.

[27] Goparaju, L., et al., Stigma, Partners, Providers and Costs: Potential Barriers to PrEP Uptake among US Women. J AIDS Clin Res, 2017. 8 (9). 